\title{
苯并咪唑类化合物的合成方法进展
}

\author{
朱观明 ${ }^{a}$ 杨柳阳 ${ }^{a}$ 崔冬梅*,a \\ (浙江工业大学药学院 杭州 310014)
}

\begin{abstract}
摘要 苯并咪唑类化合物具有抗 HIV-1、抗肿瘤、抗细胞增殖、抗寄生虫、抗炎症、抗氧化和抗癫㾋方面的生物活性, 因此这类化合物的合成备受化学家们的关注. 首次按照反应中间体将近 5 年合成这类化合物的方法分为以 $N$-亚甲基邻 苯二胺(或 $N$-亚甲基邻硝基苯胺)为中间体，以 $N$-酰基邻苯二胺为中间体和以芳基脒(胍)为中间体三类. 通过从这个角 度论述这些合成方法, 期望能够为设计出更好的合成方法提供启发.
\end{abstract}

关键词＼cjkstart苯并咪唑; $N$-亚甲基邻苯二胺; $N$-亚甲基邻硝基苯胺; $N$-酰基邻苯二胺; 芳基榺(胍); 催化剂

\section{Advances in the Synthetic Methods of Benzimidazoles}

\author{
Zhu, Guanming $^{a} \quad$ Yang, Liuyang $^{a} \quad$ Cui, Dongmei ${ }^{*, a}$ \\ (College of Pharmaceutical Science, Zhejiang University of Technology, Hangzhou 310014)
}

\begin{abstract}
Benzimidazoles possess various bioactivities, such as anti-HIV-I, antineoplastic, antiproliferation, antiparasitic and so on, hence the synthetic methods of this class of compounds have attracted much attention. In order to provide some inspiration of better synthetic method design, in this review these methods reported during past five years will be firstly classified into three types according to the reaction intermediates, namely $N$-methene $o$-diaminobenzene (or $N$-methene- $o$-nitroaniline), $\mathrm{N}$-acyl $o$-diaminobenzenes and arylamidine (aryl guanidine).

Keywords benzimidazole; $N$-methene $o$-diaminobenzene; $N$-methene- $o$-nitroaniline; $N$-acyl $o$-diaminobenzene; aryl amidine (aryl guanidine); catalyst
\end{abstract}

含有苯并咪唑环结构单元的化合物在抗 HIV-1 病 毒 $^{[1]}$ 、抗肿瘤 ${ }^{[2]} 、$ 抗细胞增殖 ${ }^{[3]}$ 、抗癫痳 ${ }^{[4]} 、$ 抗高血压 ${ }^{[5]}$ 、 抗炎症 ${ }^{[6]} 、$ 抗氧化 ${ }^{[7]}$ 和抗寄生虫 ${ }^{[8]}$ 方面表现出优秀的生物 活性, 孟江平等 ${ }^{[9]}$ 详细论述了含有该结构单元的药物在 药理活性方面的作用. 除此之外, 这类化合物还用作金 属配体. 正因为苯并咪唑类化合物具有如此多的用途, 合成这类化合物的方法层出不穷并且有很多综述从不 同角度对这些方法进行了分类论述. 李炎等 ${ }^{[10]}$ 按照反 应底物的不同将这些合成方法分为邻苯二胺与羧酸、羧 酸衍生物和醛三类论述这类化合物的合成方法. Chawla ${ }^{[11]}$ 和 Panda 等 ${ }^{[12]}$ 各自报道一篇和这种分类框架 相似的综述. 后来毛郑州等 ${ }^{[13]}$ 的综述将羒酸和羧酸的 衍生物归为一类, 并且比较具体地论述了邻苯二胺和醛 的合成方法. 此外, 这篇综述也简略地论述了过渡金属 参与合成苯并咪唑类化合物的方法. 除了这些综述外, 还有综述比较集中地论述了苯并咪唑类化合物合成方
法的某方面问题. 比如毛郑州 ${ }^{[14]}$ 和 Chawla 等 ${ }^{[15]}$ 论述了 应用于苯并咪唑环类化合物合成的各种新技术. 彭看 等 ${ }^{[16]}$ 重点论述了以天然或非天然氨基酸为底物合成苯 并咪唑类化合物的各种方法. 此外, 还有综述专门对 1,2-二取代苯并咪唑类化合物的合成方法进行了论 述 ${ }^{[17]}$. 也有综述 ${ }^{[18]}$ 重点讨论了经过重排一再环合过程合 成苯并咪唑类化合物的方法. 和非并环苯并咪唑类化合 物一样, 含有苯并咪唑环结构单元的稠环化合物也表现 出非常好的生物活性, 所以这类化合物的合成方法也吸 引了很多人的关注, 但是本文基于以下两个原因不论述 这个问题, 首先已经有综述详细地论述过这个问题 ${ }^{[19]}$, 其次本文关注的是苯并咪唑环基本结构单元的构建，而 多数构建并环苯并咪唑的方法都是在这个基本结构单 元上开始的. 综合分析这些综述, 我们发现还没有综述 按照合成过程中不同的中间体归纳这类化合物的合成 方法. 于是我们决定从更加能够反映合成方法的中间体

\footnotetext{
*E-mail: cuidongmei@zjut.edu.cn; 892534827@qq.com

Received October 13, 2013; revised November 16, 2013; published online November 28, 2013.

Project supported by the Natural Science Foundation of Zhejiang Province (No. Y4100558).

浙江省自然科学基金(No. Y4100558)资助项目.
} 
出发, 对这类化合物的合成方法(主要是 2005 年至今的 文献)进行了分析整合, 并且把它们按照中间体分为三 类, 即 $N$-亚甲基邻苯二胺(或 $N$-亚甲基邻硝基苯胺)为中 间体的合成方法, $N$-酰基邻苯二胺为中间体的合成方法 和芳基榺(胍)为中间体的合成方法. 通过这种新的分类 归纳方法, 我们期望能够从中获得某些启发, 从而设计 出更好的合成方法.

\section{1 以 $N$-亚甲基邻硝基苯胺或 $N$-亚甲基邻苯二 胺为中间体}

虽然以 $N$-亚甲基邻硝基苯胺为中间体的合成方法 也有报道，但是本文在这部分主要论述以 $N$-亚甲基邻 苯二胺为中间体的合成方法.

$N$-亚甲基邻苯二胺衍生物 $\mathbf{1}$ 在氧化条件下环合合成 苯并咪唑类化合物是一直以来研究最多的方法. 中间体 1 通过邻苯二胺衍生物和醛脱水缩合得到, 并且和苯并 咪唑啉 2 互变异构. 1 和 2 的热动力学稳定性是不同的, 因为在探测反应中间体的实验中只发现了 1 , 推测可能 2 很活泼, 所以一旦形成就转变为其他产物, 因此没有 被仪器探测到 ${ }^{[20]} .1$ 或 2 在氧化剂的作用下脱氢得到苯 并咪唑环结构单元(Scheme 1).
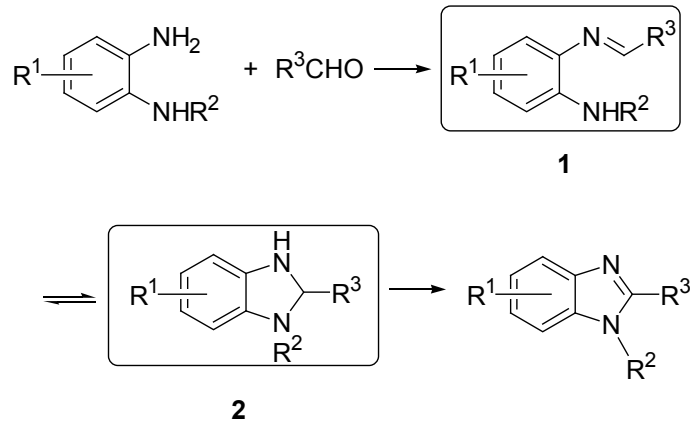

Scheme 1

当 $\mathrm{R}^{2}$ 为 $\mathrm{H}$ 时, $\mathbf{1}$ 和另一份醛反应，得到 $N, N^{\prime}$-二亚甲 基邻苯二胺衍生物 $\mathbf{3}$, 中间体 3 经过 [1,3] $\delta$ 迁移得到 1,2二取代苯并咪唑类化合物. 在这里, 由于中间体 1 同时 存在于 Schemes 1 和 2 所示的两种反应历程中, 因此反 应可能得到两种产物: 2-取代苯并咪唑和 1-取代亚甲基2-取代苯并咪唑. 由于这两类产物具有重要的应用价 值, 人们也在探索如何控制反应选择性地合成这两类化 合物. 有综述论述过反应选择性的问题, 该综述 ${ }^{[21]}$ 总结 了 2011 年前文献报道过的合成 1,2-二取代苯并咪唑类 化合物的方法, 并且指出以芳香醛和邻苯二胺为原料合 成 1,2-二取代苯并咪唑类化合物的方法存在选择性不好 的问题.

目前, 由于全氟取代溶剂作为反应介质引入到有机 合成上，化学家们能够很好地控制反应选择性，对于大
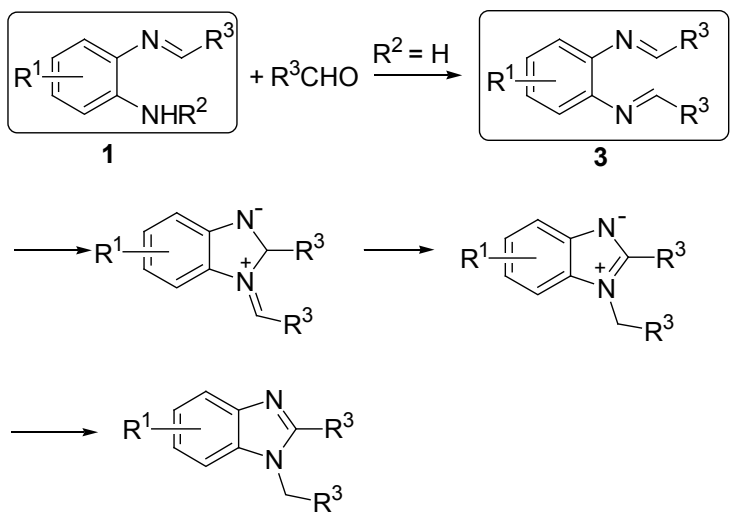

\section{Scheme 2}

部分醛，这种方法都可以高收率地得到 1,2-二取代苯并 咪唑类化合物(Eq. 1) ${ }^{[22]}$. 由于已经有综述对这个问题进 行详尽的讨论，并且这个反应的中间体是二亚胺，而这 部分要论述的中间体是 $N$-亚甲基邻苯二胺或 $N$-亚甲基 邻硝基苯胺，并且经过这类中间体合成 1,2-二取代苯并 咪坐类化合物的合成方法和下文将要讨论的方法相同, 只要使用适当的底物就可以合成这类化合物，所以不在 本文重复讨论.

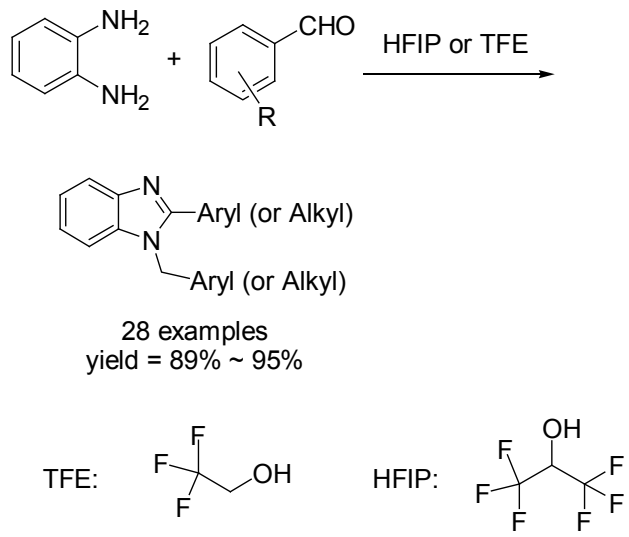

$\mathrm{R}=\mathrm{H}, 4-\mathrm{CH}_{3}, 4-\mathrm{OCH}_{3}, 4-\mathrm{N}\left(\mathrm{CH}_{3}\right)_{2}, 4-\mathrm{Cl}, 4-\mathrm{Br}, 4-\mathrm{OH}, 4-\mathrm{CF}_{3}$ 4-CN, 4- $\mathrm{NO}_{2}$, 4-OCH${ }_{2} \mathrm{Ph}-4-\mathrm{OCOPh}$, 4-OCOBu- $t$, 4-OBoc, 4-TBMS, 3,5-( $\left(\mathrm{OCH}_{3}\right)_{2}, 3,4-\left(\mathrm{OCH}_{3}\right)_{2}$

综合分析已经报道过的合成方法, 多数合成方法在 催化剂或氧化剂体系下进行，反应主要产物是 2-取代苯 并咪唑类化物，次要产物是 1-取代亚甲基-2-取代苯并 咪唑类化合物. 以下将以催化剂/氧化剂体系对已经报 道的方法进行分类论述.

\section{1 单独氧化剂参与的合成方法}

中间体 1 在氧化剂作用下得到苯并咪唑类化合物 (Eq. 2).

溴化二甲基溴化锍(BDMS)是一种廉价易得的亮黄 色固体化合物，可以用于催化多种化学反应，也可以作 为溴代试剂参与化学反应. 2007 年 Das 等 ${ }^{[23]}$ 报道以 1 equiv.的溴化二甲基溴化锍参与苯甲醛和邻苯二胺反应 


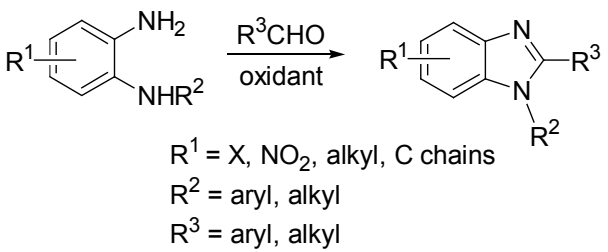

得到 2-芳基苯并咪唑, 收率为 72\% 91\%. 在这种方法 中溴化二甲基溴化锍作为氧化剂直接参与反应，同时它 在空气中形成的 DMSO 也参与氧化中间体.

同样作为具有氧化性的含溴化合物, PBBS 或 $\mathrm{TBBDA}^{[24]}$ 和乌托品溴也被用于合成苯并咪唑类化合物. 2012 年 Khosravi 等 ${ }^{[25]}$ 以 $10 \mathrm{~mol} \%$ 乌托品溴参与邻苯二 胺和各种醛的反应(Eq. 3), 选择性地得到 2-芳基苯并咪 唑. 该反应在室温条件下反应 5 6 h, 收率为 $70 \%$ $80 \%$; 在引入微波技术后，大大缩短了反应时间并且反 应收率和室温收率相当. 在反应过程中作为溴供体的乌 托品溴可以活化醛上的羰基, 而本身具有的氧化性可以 把苯并咪唑啉氧化为产物.<smiles>[R][X]O[N+]([O-])([O-])O[Na]</smiles><smiles>CCCCCCC(C)N(CC)S(=O)(=O)c1cccc(S(=O)(=O)N(Br)C(C)C(C)(C)N(Br)S(=O)(=O)c2cccc(S(=O)(=O)N(Br)Br)c2)c1</smiles>

早在 1971 年 Smith 等 ${ }^{[26]}$ 把邻苯二胺和苯甲醛暴露在 空气中反应，若干天后，得到的反应产物为化合物 5 和 6 的 $1: 1$ (物质的量的比)混合物以及化合物 4 (Scheme 3).

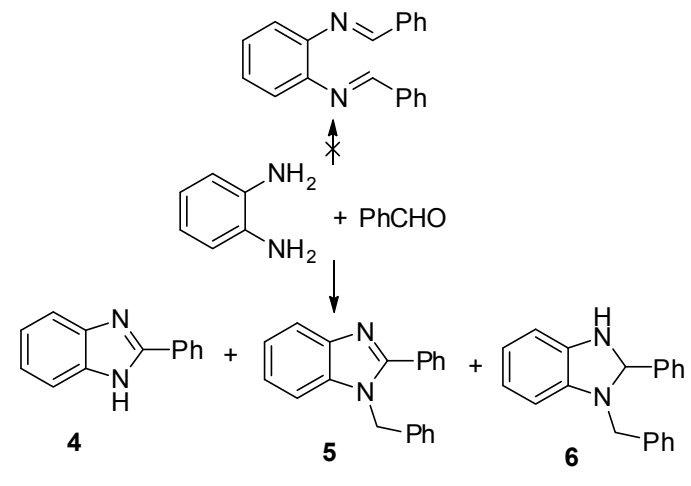

Scheme 3

无催化存在下用空气作氧化剂合成苯并咪唑类化 合物是合成这类化合物的绿色方法. 2005 年 Lin 等 ${ }^{[27]}$ 报 道在没有催化剂存在下用空气中氧气氧化合成 2-芳基
苯并咪唑. 在空气存在下，回流或在 $100{ }^{\circ} \mathrm{C}$ 下反应 $4 \mathrm{~h}$ 得到目标产物. 当以二氧六环为溶剂, 产物收率得到了 提高. 尽管对有些底物反应时间太长，该合成反应因为 没有使用重金属催化剂而具有一定优越性.

2011 年, Panda 等 ${ }^{[28]}$ 在以水为溶剂的非均相体系中 没有催化剂存在下升温到 $100{ }^{\circ} \mathrm{C}$ 反应 $2 \mathrm{~h}$, 各种取代芳 香醛高收率地得到了苯并咪唑类化合物. 有趣的是, 在 2005 年 Lin 等 ${ }^{[27]}$ 所报道合成方法的条件优化中，以水作 为溶剂在 $100{ }^{\circ} \mathrm{C}$ 下即使反应 $4 \mathrm{~h}$, 反应收率也只有 $58 \%$. 此外, Zhang 等 ${ }^{[29]}$ 也以甲苯为溶剂暴露在空气中室温下 合成 2-环丙基、2-戊基以及环已基苯并咪唑，这种方法 对烷基甲醛反应收率很高，对芳香醛却不适用. 类似地， Chen 等 ${ }^{[30]}$ 将反应暴露在空气中并且以乙醇为溶剂室温 下合成苯并咪唑类化合物, 有意思的是, 和 Zhang 等所 报道方法的适用范围不同，这种方法适用于绝多数芳香 醛.

硝基化合物也被用作氧化剂参与合成苯并咪唑类 化合物, 并且在引入负载技术微波和超声之后, 很好地 克服了反应温度高、反应时间长的不足(Eq. 4) ${ }^{[31]}$.

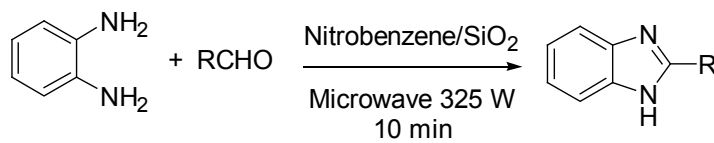

除了硝基苯以外，2,3-二氯-5,6-二氰基-1,4-苯醌 $(\mathrm{DDQ})^{[32]}$ 和过氧单磺酸钾 (oxone) $)^{[33]}$ 也用于合成苯并咪 唑类化合物. 作为在有机合成中具有多种用途的氧化 剂 $^{[34]}$ ，单质碘也用于苯并咪唑类化合物合成 ${ }^{[35]}$.

上面的合成方法都使用等量的无机或有机氧化剂, 这不仅浪费试剂, 而且产生了大量的副产物, 导致原子 经济性很低, 所以有必要将氧化剂当量参与的反应改造 成催化量就能进行的反应. 经过不断的尝试和总结，人 们发现变价金属(或复合物)可以和氧化剂组成氧化催化 体系, 并且成功地应用于催化合成苯并咪唑类化合物.

\section{2 变价金属(或复合物)和氧化剂体系}

由于具有一定的氧化性，等量的醋酸铜曾用来氧化 合成苯并咪唑类化合物. 为了实现催化量催化反应, Sharghi 等 ${ }^{[36]}$ 用 Salen 配体 7 和铜(II)组成的络合物作为 非均相催化剂在初始氧化剂存在下催化合成苯并咪唑 类化合物. 这种方法不仅适用于芳香醛和杂环醛，也适 用于酸敏感和高位阻的醛. 在反应过程中, $\mathrm{Cu}(\mathrm{II})$ 和苯并 咪唑啉 8 作用得到 $\mathrm{Cu}(\mathrm{I})$ 和自由基中间体 $\mathbf{9}$, 自由基中间 体 9 在氧气或者羟基自由基作用下得到产物和 $\mathrm{HOO}^{-}$或 者水, 而 $\mathrm{Cu}(\mathrm{I})$ 在反应过程中产生的双氧水和 $\mathrm{HOO}^{-}$的 作用下再生为铜(II). 值得注意的是, 除了 $\mathrm{O}_{2}$ 作为初始 氧化剂外, 反应过程中产生的双氧水也作为初始氧化剂 
能够促进反应进行，实验同时也证明，把双氧水加入到 反应体系能够加快反应速率(Scheme 4).

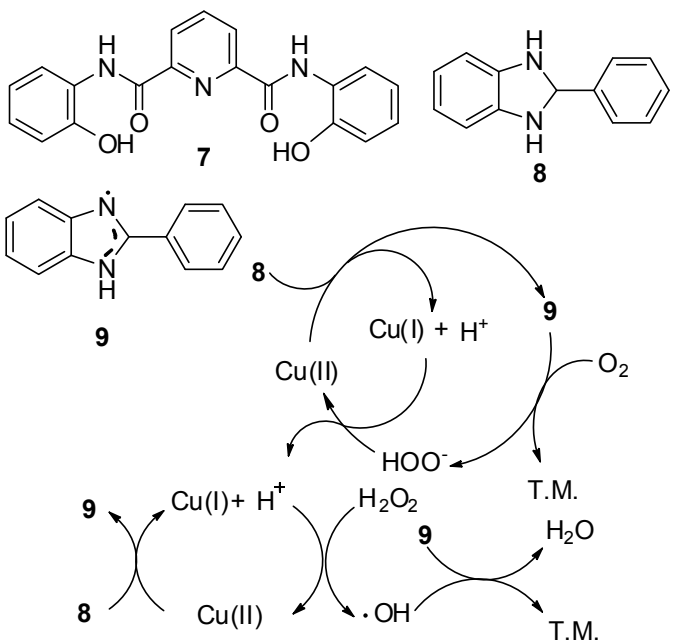

Scheme 4

和 $\mathrm{Cu}(\mathrm{II}) / \mathrm{Cu}(\mathrm{I})$ 电对相似, 作为可逆电对, $\mathrm{Ce}(\mathrm{IV}) /$ $\mathrm{Ce}(\mathrm{III})$ 高价铈盐和初始氧化剂双氧水也组成催化体系应 用于催化合成苯并咪唑类化合物(Scheme 5) ${ }^{[37]}$. 反应适 用性实验表明给电子和吸电子取代基取代的芳香醛以 及 $\alpha, \beta$-不饱和醛都得到 $92 \%$ 以上的收率. 在这个反应中, $\mathrm{Ce}(\mathrm{IV})$ 作为直接氧化剂参与反应, 因为仅仅只有双氧水 参与反应时, 即使反应 $10 \mathrm{~h}$ 收率也很低. 另外脂肪醛和 呋喃醛不适用于这种方法.

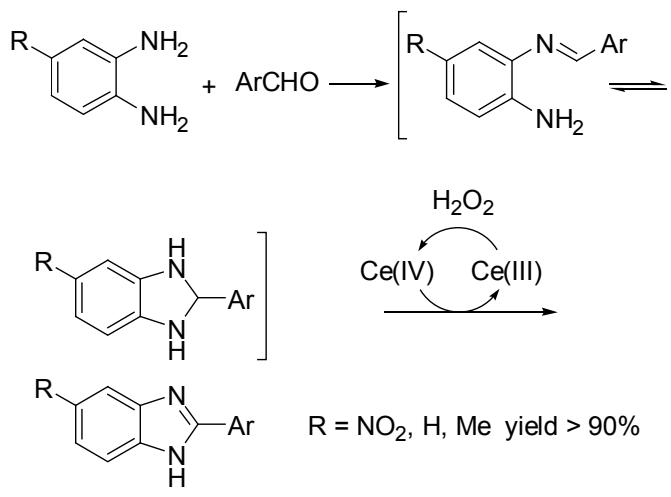

Scheme 5

作为变价金属, 离子状态存在的 Co 和双氧水体系 也用于催化合成苯并咪唑类化合物 ${ }^{[38]}$. 此外, Sharghi 等 ${ }^{[39]}$ 设计了 Co 金属/氧化剂体系. 和之前 Sharghi 等所 报告的方法不同，这种方法以负载到活性炭上的 Salen-Co 复合物为催化剂, 并且适用范围更广, 除了芳 香醛以外, 脂肪醛和杂环醛都可以获得很好的收率. 这 个机理和 Salen-Cu 的催化机理(Scheme 4)相似.

$\mathrm{Fe}(\mathrm{III}) / \mathrm{Fe}$ (II)还原电对和氧气组成的催化体系也用 来催化合成苯并咪唑类化合物 ${ }^{[40]}$. 铁(III)和卟啉配体组
成的复合物具有催化有机物氧化的作用 ${ }^{[41]}$. Sharghi 等 ${ }^{[39]}$ 成功地用铁(III)卟啉催化利用空气中的氧气合成苯 并咪唑环类化合物. 在这种方法中卟啉配体非常重要, 当只有三氯化铁存在时，即使反应 $24 \mathrm{~h}$ 收率也只有 $20 \%$. 这种合成方法的机理为: 铁(III)卟啉(I)从 8 夺得电子形 成 $\mathrm{Fe}$ (II)卟啉(II)和自由基 $\mathbf{9}, \mathrm{Fe}$ (II)卟啉(II)在氧气作用下 经过 III 和 IV 得到 $\mathrm{Fe}(\mathrm{IV})=\mathrm{O}$ 卟啉 $(\mathbf{V}), \mathbf{V}$ 再从 9 夺得一 个电子, 自身还原为 $\mathrm{Fe}(\mathrm{III})$ 卟啉(I), 同时得到目标产物 (Scheme 6).

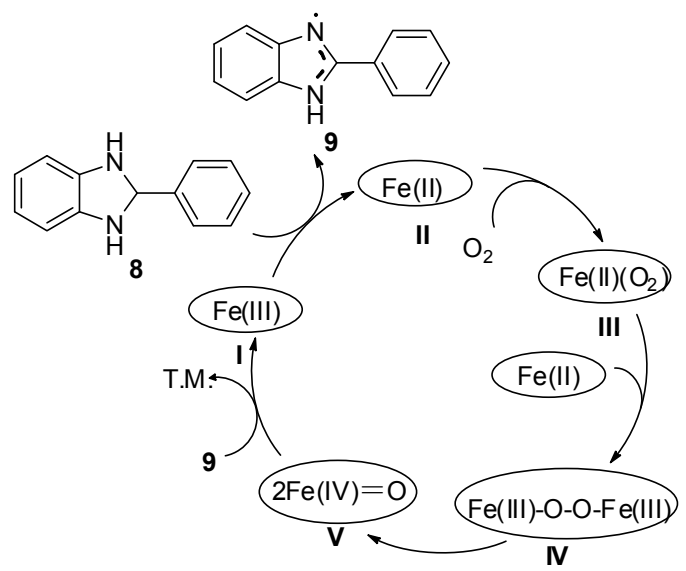

Scheme 6

此外，新技术诸如负载和超声技术以及生物酶技术 被引入到苯并咪唑类化合物的合成上.

2010 年 Abdollahi Alibeik 等 ${ }^{[42]}$ 将氯化铁分散于多聚 苯胺并且用来催化合成 2-芳基苯并咪唑类化合物. 这个 方法反应收率在 70\% 97\%之间，适用于杂环醛和芳香 醛以及不饱和醛. 同年, Fazlinia 等 ${ }^{[43]}$ 用负载在 $\mathrm{SiO}_{2}$ 的氯 化铁作为催化剂, $\mathrm{H}_{2} \mathrm{O}_{2}$ 作为初始氧化剂合成苯并咪唑类 化合物. 在这种方法中, 铁(III)和 $\mathrm{H}_{2} \mathrm{O}_{2}$ 形成的高价铁是 $N$-亚甲基邻苯二胺的直接氧化剂.

超声技术也被应用于这类化合物的合成. 2011 年， Chen 等 ${ }^{[44]}$ 将氯化铁负载到氧化铝上, 然后在超声作用 下催化合成苯并咪唑类化合物. 由于使用了负载和超声 技术，大大降低了反应温度和缩短了反应时间.

漆酶(Laccases) 是一种结合多个铜离子的蛋白质， 可以在温和的条件下以氧气作为氧化剂选择性地氧化 多种底物. 2011 年 Leutbecher 等 ${ }^{[45]}$ 以氧气作为初始氧化 剂用这种酶催化邻苯二胺和芳香醛反应合成了苯并咪 唑类化合物(Scheme 7).

\section{3 自由基催化剂和氧化剂体系}

$N$-羊基邻苯二甲酰亚胺(NHPI)在反应过程中形成 $N$-氧自由基参与烃类化合物在空气中的氧化. 这类化合 物和 $\mathrm{Co}(\mathrm{AcO})_{2}$ 组成的体系用来催化合成苯并咪唑啉类 化合物 ${ }^{[46]}$. 研究表明, 仅加入 NHPI 参与反应, 反应需 


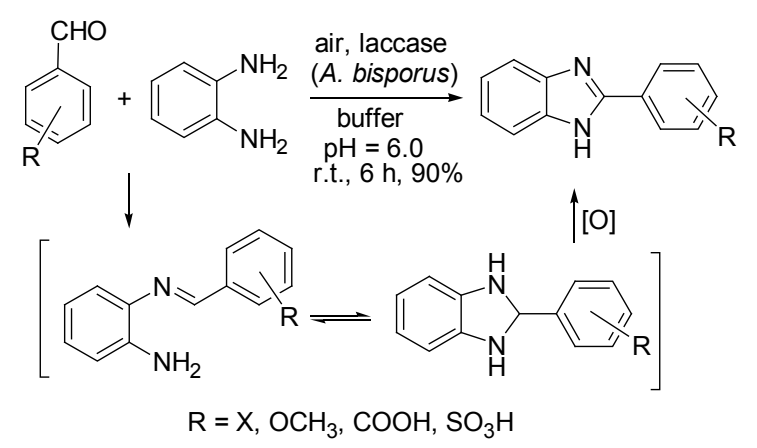

Scheme 7

在 $100{ }^{\circ} \mathrm{C}$ 下才能进行, 加入少量醋酸钴后反应可以在 室温下进行. 取代基电性效应对反应时间影响很大, 供 电子取代基有利于反应进行, 吸电子取代基对反应不 利, 会使反应时间延长, 有些强吸电子取代基甚至需要 $6 \mathrm{~d}$ 才能反应完全.

TEMPO 是一类氮氧化合物, 它的氧原子上带有一 个孤对电子, 它可以作为自由基催化剂和氧气组成的体 系催化合成苯并咪唑类化合物 ${ }^{[47]}$. 这种方法在 $101 \mathrm{kPa}$ 氧气中, 以二甲苯为溶剂 $120{ }^{\circ} \mathrm{C}$ 反应 $7 \sim 11 \mathrm{~h}$, 收率为 $89 \% \sim 91 \%$. 它的机理是: 在氧气存在的条件下, 苯亚 甲基邻苯二胺(12)氨基上的 $\mathrm{C}-\mathrm{H}$ 键在 10 作用下发生均 裂, 形成自由基 13, 13 环合形成苯并咪唑啉自由基 14, 该自由基在 10 和氧气的参与下进一步氧化为目标产物 (Scheme 8).

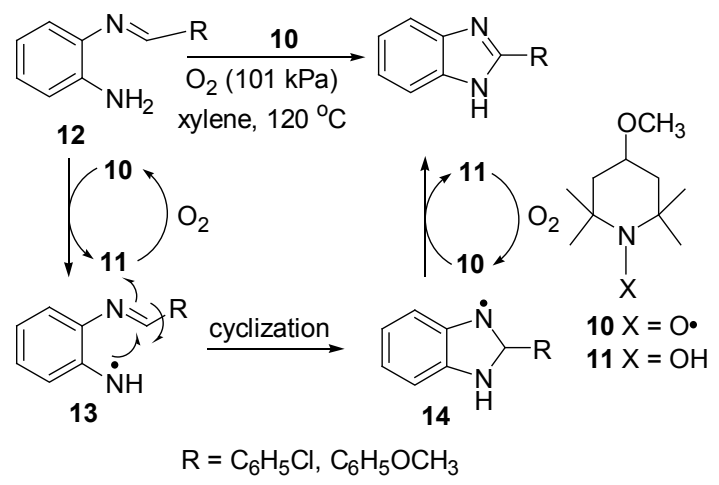

Scheme 8

\subsection{Lewis 酸或杂多酸、质子酸和氧化剂体系}

Lewis 酸与杂多酸一直被应用于很多有机化学反 应，这类化合物也被用来催化苯并咪唑类化合物的合 成. 2009 年 Rostamizadeh 等 ${ }^{[48]}$ 把含有结晶水的二氯氧锆 沉积在蒙脱土-K10 上得到催化剂, 然后用来催化合成 苯并咪唑类化合物. 这种反应由于以水为溶剂并且反应 时间短，因此具有一定优势. 同年 Aliyan 等 ${ }^{[49]}$ 以浸渍磷 酸锆的磷铇酸作为非均相催化剂催化合成苯并咪唑.

由于具有适度的铝含量, 酸度和独特的比表面积以 及特异的孔体积, AlKIT-5(10)被用来催化合成苯并咪唑
类化合物 ${ }^{[50]}$. 这个方法适用于芳香醛和杂环醛以及脂 肪醛，反应收率为 $70 \% \sim 90 \%$.

以锌-氯交换的 K10-蒙脱土是一种优良的 Lewis 酸, Dhakshinamoorthy 等 ${ }^{[51]}$ 以这种 Lewis 酸酸催化合成芳基 苯并咪唑类化合物. 这种合成方法机理和锌-氯交换 K10-蒙脱土上的锌 Lewis 酸位点密切相关. 由于在晶体 结构中含有众多的 Lewis 酸或 Lewis 碱位点, 各种金属 氧化物也经常和氧化剂组合在一起催化合成苯并咪唑

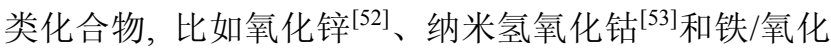
铈一氧化锆纳米颗粒 ${ }^{[54]}$. 我们课题组在空气存在的条件 下用金属氧化锌催化邻苯二胺和芳香醛反应合成苯并 咪唑类化合物, 芳香醛上苯环不同的取代基对产物的分 布具有一定的影响 ${ }^{[5]}$.

除了 Lewis 酸可以催化苯并咪唑类化合物的合成, 质子酸也可以催化合成这类化合物. 2007 年 Han 等 ${ }^{[56]}$ 以 $20 \mathrm{~mol} \%$ 对甲苯磺酸为催化剂催化合成 2-取代苯并咪 唑, 该催化剂体系不适用于脂肪醛. 氧气被认为是氧化 苯并咪唑啉的氧化剂. 2007 年 Chakrabarty 等 ${ }^{[57]}$ 将对甲 苯磺酸负载到硅胶上. 尽管这个催化体系催化合成 2-芳 基苯并咪唑的选择性不好, 但是 2-芳基苯并咪唑和 1,2二取代苯并咪唑的总收率为 78\% 99\%。这种方法对于 某些芳香醛表现出一定的选择性，对于吸电子基团取代 的芳香醛(对氧基苯甲醛和间硝基苯甲醛)可以选择性地 得到 1,2-二取代苯并咪唑, 而对硝基苯甲醛完全得到 2芳基苯并咪唑. 醛和单 $N$-烷基取代邻苯二胺在这个催 化体系催化下得到 77\% 82\%的 1,2-二取代苯并咪唑.

2009 年 Mohammaidi 等 ${ }^{[58]}$ 用负载型的 Caro's 酸在 加热回流条件下催化合成苯并咪唑化合物. 在这个催化 体系中, 氧气将 $N$-亚甲基邻苯二胺氧化为 2 -取代苯并 咪唑.

由于磷氧双键上的氧和羟基上的质子可以双重催 化活化羰基和氨基, 环磷酸 ${ }^{[99]}$ 可以催化邻苯二胺和醛 反应快速成为 $N$-亚甲基邻苯二胺中间体, 随即在催化 剂作用下，这个中间体迅速环合-氧化生成苯并咪唑类 化合物.

\section{5 离子液体或有机酸盐以及无机盐和氧化剂体系}

2007 年 Srinivas 等 ${ }^{\left[{ }^{[0]}\right.}$ 用多聚苯胺的硫酸盐不仅在室 温下成功催化合成了苯并咪唑类化合物，而且可以顺利 地回收催化剂. 作为有机酸盐, 醋酸铵也被用于催化合 成苯并咪唑类化合物. 2008 年 Sharghi 等 ${ }^{[6]}$ 在无水乙醇 中以等量的醋酸铵升温到 $75{ }^{\circ} \mathrm{C}$ 催化邻苯二胺和取代苯 甲醛的缩合反应，反应适用性研究表明，这种方法对于 大多数醛可以得到 2-芳基苯并咪唑, 但是某些醛仍然会 得到 1,2-二取代苯并咪唑. 此外, 咪唑类离子液体 ${ }^{[62]}$ 和 氨基酸类离子液体 ${ }^{\left[{ }^{[3]}\right.}$ 也应用于苯并咪唑类化合物的合 
成.

2012 年 Lei 等 ${ }^{[64]}$ 以 $3 \mathrm{~mol} \%$ 的维生素 B1 的盐酸盐为 催化剂, 在 DMF 中室温下催化合成芳基苯并咪唑类化 合物. 很有趣的是, 反应物的投料比对反应产物种类分 布影响非常大, 当邻苯二胺和醛的比例为 $1: 3$ 时, 反应 产物为 1,2-二取代苯并咪唑; 当邻苯二胺和醛的比例为 $1: 1$ 时, 反应产物为 2-芳基取代苯并咪唑.

作为长链烃磺酸盐, 庚烷基磺酸钠 ${ }^{[65]}$ 和十二烷基 磺酸钠 $(\mathrm{SDS})^{[66]}$ 被用来催化苯并咪唑类化合物. 和 Lei 报道的方法相类似, 当以 SDS 催化合成苯并咪唑类化 合物时, 醛和邻苯二胺的比例对产物的种类分布影响很 大, 当醛/邻苯二胺的物质的量为 $1: 2$, 反应高收率得 到 1-芳亚甲基-2-芳基苯并咪唑; 当醛/邻苯二胺的物质 的量为 $1: 1$ 并且氧化剂存在时, 反应选择性地得到 2芳基苯并咪唑. 不足的是该反应体系不适用于脂肪醛. 这种方法的机理是: SDS 在水相形成胶团, 并且把反应 物包裹在里面, 从而可以促进脱水形成 $N$-亚甲基邻苯 二胺中间体，同时十二烷基磺酸作为质子酸活化苯甲醛 上的羰基.

过长反应时间和太高反应温度对有些反应是不利 的, Molander 等 ${ }^{[67]}$ 为了合成含苯基硼酸盐的苯并咪唑环 类化合物, 以氧气作为氧化剂, 而且将 $\mathrm{KH}_{2} \mathrm{~F}$ 引入到反 应体系, 从而使反应温度降低到 $40{ }^{\circ} \mathrm{C}$, 克服了在通常 氧化条件下反应时间长温度太高而容易发生脱嗍副反 应的缺点.

以上所述是以醛作为底物和邻苯二胺类衍生物反 应合成目标产物的方法, 但是醛不稳定而且价格相对不 便宜, 所以人们希望用便宜稳定的醇替代醛参与反应. 通过原位底物 $\mathrm{C}-\mathrm{H}$ 活化构建化学键的策略为这种替代 带来了曙光, 这种策略通过催化脱氢氧化将底物活化, 然后进行新化学键的构建步骤 ${ }^{[68]}$, 从而大大提高了反 应的原子经济性. 在这种新策略的指导下, Bala 等 ${ }^{[69]}$ 用 稳定便宜的醇替代容易被氧化的醛和邻苯二胺在 $\mathrm{PcFe}$ (Iron Phthalocyanine) 反应合成苯并咪唑类化合物. 与脂 肪族醛相比较, 芳香醛更加适用于这种方法. 另外, 与 以上所论述的方法不同, 这种方法所需要的氧化剂不是 空气中氧气, 因为这些反应都是在 $\mathrm{N}_{2}$ 保护下进行. 很有 趣的是, 尽管 $\left[\mathrm{PcFeH}_{2}\right]$ 可以还原亚胺, 但是在这种合成 方法中 $N$-亚甲基邻苯二胺或苯并咪唑啉能够很好地优 先被 $[\mathrm{PcFe}]$ 氧化为目标产物, 避免被 $\left[\mathrm{PcFeH}_{2}\right]$ 还原为 $N$ 苠基邻苯二胺(Scheme 9).

除了在金属催化剂存在下用催化脱氢氧化将底物 活化, 半导体材料也用于底物活化. 负载 $\mathrm{TiO}_{2}$ 纳米颗粒 的铂在紫外线和太阳光作用下发生光诱导电子转移, 在 铂的内部形成很多自由电子和空穴, 这些电子和空穴构

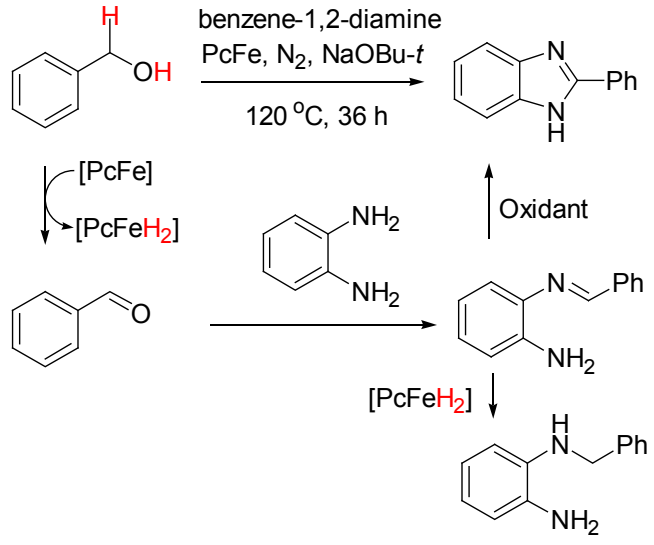

Scheme 9

成还原和氧化的微环境, 铂的这种性质被用于催化有机 合成反应。在这种催化剂存在下， $N$-芳基邻硝基苯胺和 醇经过 $N$-亚甲基邻苯二胺中间体合成苯并咪唑类化合 物(Scheme 10) ${ }^{[70]}$.

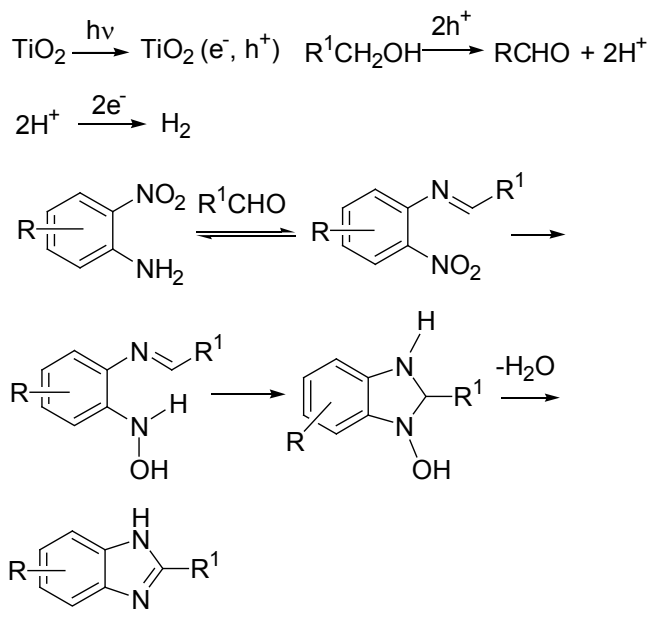

Scheme 10

\section{2 以 $N$-酰基邻苯二胺为中间体}

作为中间体， $N$-酰基邻苯二胺衍生物(酰胺类化合 物)在一定条件下经过脱水一环合也可以合成苯并咪唑 类化合物. 早在 20 世纪 50 年代, Rope 等 ${ }^{[71]}$ 连续报道了 以翔酸和邻苯二胺为原料加热合成 2-卤代苯基苯并咪 唑类化合物(Scheme 11).

由于具有促进中间体的羰基氧和氨基上的氢发生 脱水反应作用，各种脱水剂也被应用于以上反应. 2004 年, Clemens 等 ${ }^{[72]}$ 以 PyBOP 为脱水剂在条件 $\mathrm{a}$ 下合成 2取代苯并咪唑. 2012 年, Wen 等 ${ }^{[73]}$ 以 $\mathrm{T} 3 \mathrm{P}$ 为脱水缩合剂 在条件 b 下合成 2-取代苯并咪唑. 多聚磷酸也被用于脱 水来合成这类化合物, 但是没有解决反应温度高 $(>180$ $\left.{ }^{\circ} \mathrm{C}\right)$ 和反应时间长的缺点(Eq. 5 $)^{[74]}$. 


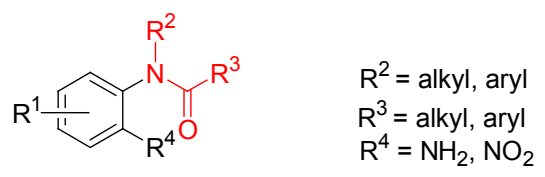

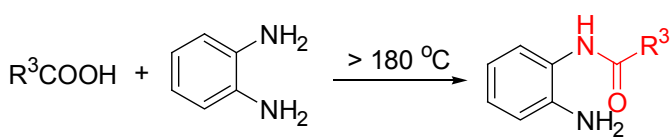

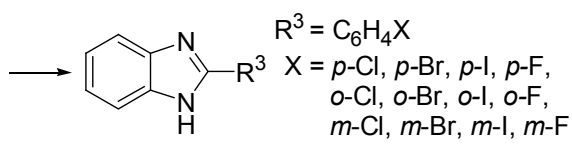
yield $<21 \%$

\section{Scheme 11}

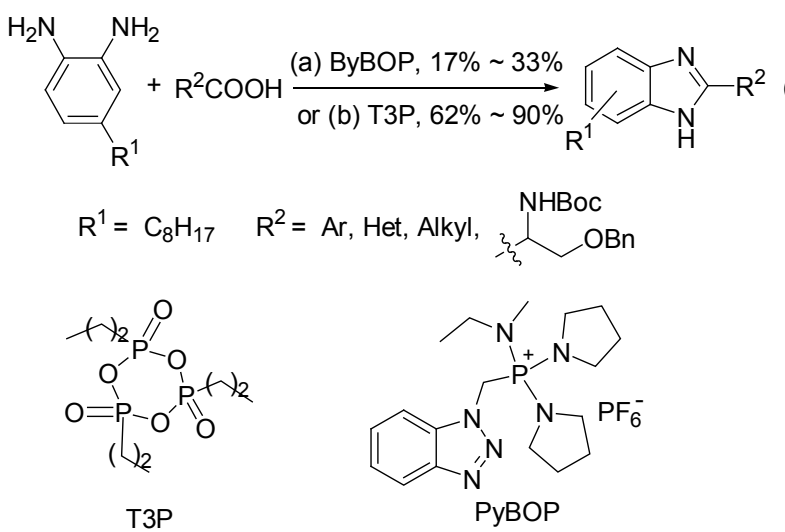

为了降低反应温度, Algul 等 ${ }^{[75]}$ 在多聚磷酸存在下 用微波加热合成 12 个苯并咪唑类化合物, 这个方法和 传统加热方法相比, 收率增加了 $3 \% \sim 113 \%$, 反应时间 减少了 $95 \% \sim 98 \%$ (Eq. 6). 最近, Khattab 等 ${ }^{[76]}$ 也报道了 类似合成方法.

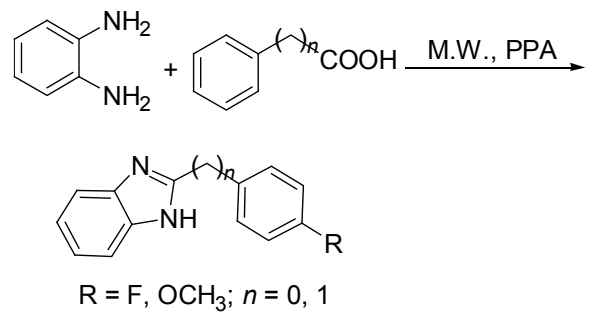

作为酰化试剂, 各种羧酸衍生物也被应用于合成 $N$-酰基邻苯二胺. 非均相固体催化剂具有催化剂易回收 和催化活性高的特点. $\mathrm{KF} / \mathrm{Al}_{2} \mathrm{O}_{3}$ 作为有效的固体非均相 碱性催化剂, Bahadori 等 ${ }^{\left[{ }^{[7]}\right.}$ 用 $\mathrm{KF} / \mathrm{Al}_{2} \mathrm{O}_{3}$ 在室温下催化邻 苯二胺与酰氯, 酸䣶和酯反应合成了一系列苯并咪唑类 化合物，这种方法具有收率高反应时间短的特点. (Scheme 12).

由于比一般的酰胺更活泼, Weinerb 酰胺也被用作 为酰化试剂和邻苯二胺衍生物反应得到 $N$-芳酰基邻苯 二胺中间体, 中间体在三氟化硼的乙醚溶液催化下脱水 环合得到苯并咪唑(Eq. 7) ${ }^{[78]}$.

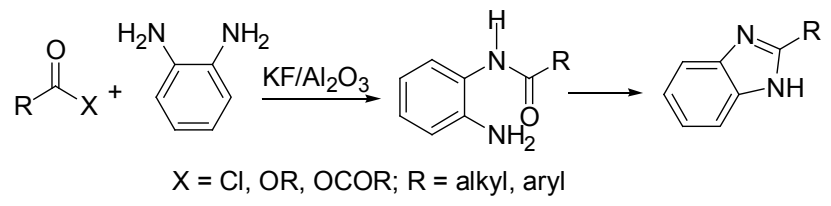

Scheme 12

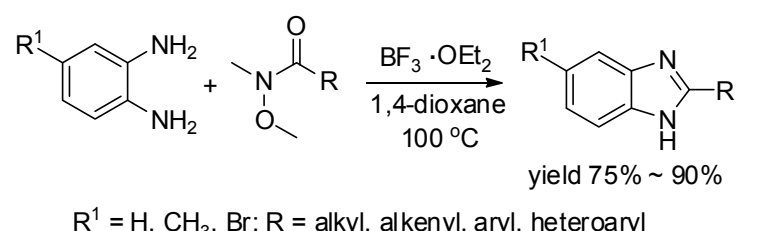

除了用羧酸或羧酸的衍生物合成 $N$-酰基邻苯二胺 中间体，也可以用卤代苯作为起始原料在钯催化下合成 这种中间体，这种中间体经过脱水一环合得到目标产物. 1993 年, Perry 等 ${ }^{[79 a]}$ 用卤代苯合成了 $N$-酰基邻苯二胺中 间体，这个中间体进一步脱水环合为苯并咪唑环. 比钯 便宜的铜催化剂也用于合成这类中间体的类似物，通过 这种中间体 She 等 ${ }^{[796]}$ 合成了一系列 2-烷基(芳基)亚甲 基- $1 H$-苯并咪唑(Scheme 13).

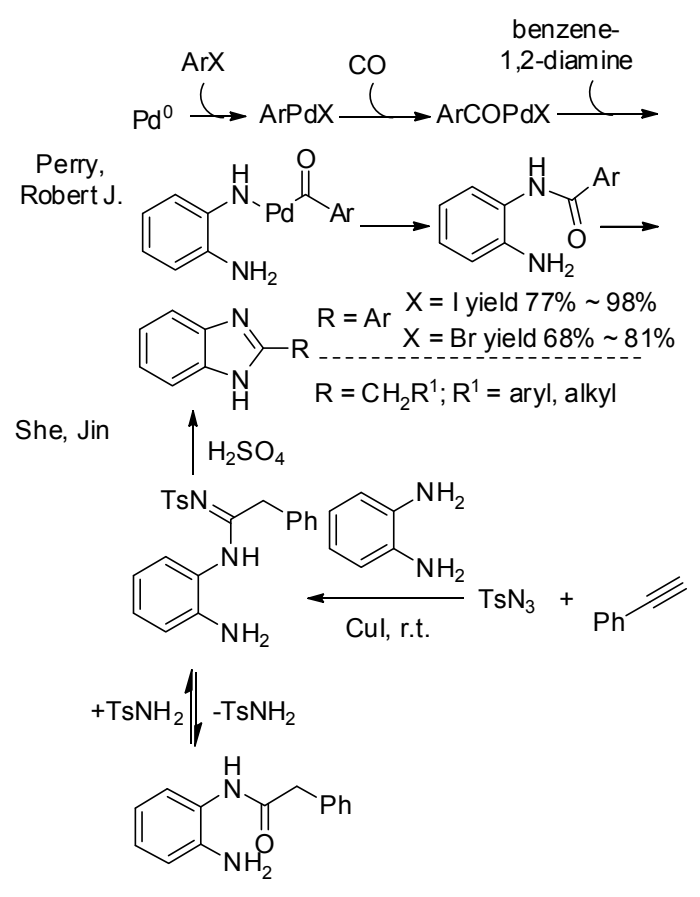

Scheme 13

邻硝基苯胺也可以取代邻苯二胺作为反应原料, 它 在微波和氯化亚锡作用下原位还原-缩合得到 $N$-烷基 (芳基)酰基邻苯二胺中间体，中间体脱水环合得到苯并 咪唑类化合物 ${ }^{[80]}$. 此外, Mamedov 等 ${ }^{[81]}$ 报道了一种通过 重排合成苯并咪唑类化合物的方法，他们将喹唑啉酮衍 生物和 1,2-二氨基丁二腈在醋酸下回流反应 $2 \mathrm{~h}$, 反应 过程中形成 $N$-酰基邻苯二胺，中间体脱水得到产物 
(Scheme 14). 这类杂环化合物通过重排再环合合成苯 并咪唑类化合物的方法也形成了区别于以羧酸或羧酸 衍生物为底物的独特合成方法, 在相关的综述 ${ }^{[82]}$ 中有 对这个问题的详细论述.

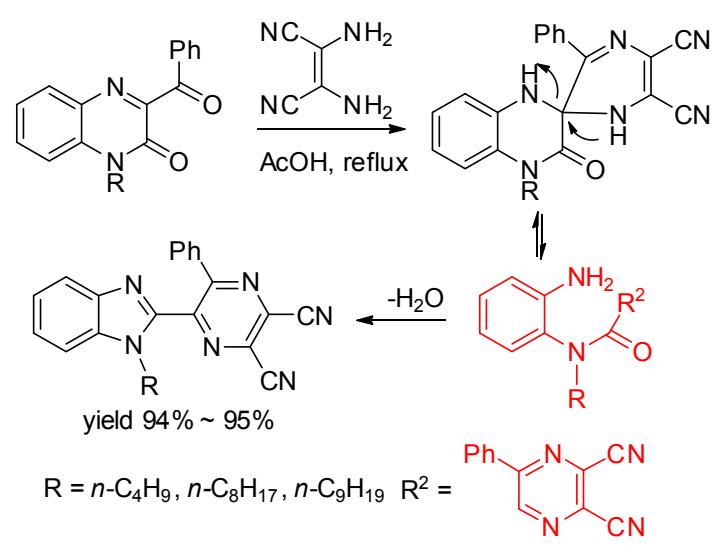

\section{Scheme 14}

\section{3 以芳基缕或芳基胍为中间体}

在众多苯并咪唑类化合物的合成方法中, 以芳基脒 或芳基胍(Chart 1)为中间体的合成方法由于方兴未艾的 $\mathrm{C}-\mathrm{N}$ 键构建方法的研究, 它的适用范围变得更广条件 更加温和.

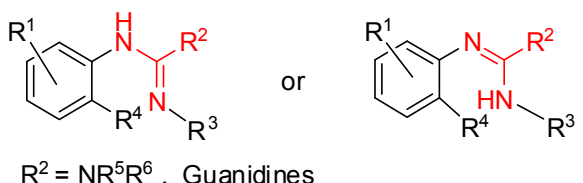

\section{Chart 1}

1999 年, Esser 等 ${ }^{[83]}$ 报道间硝基苯胍在碱性条件下 环合得到硝基苯并咪唑(Scheme 15).<smiles>[R]NC(=NO[R])Nc1cccc([N+](=O)[O-])c1</smiles>

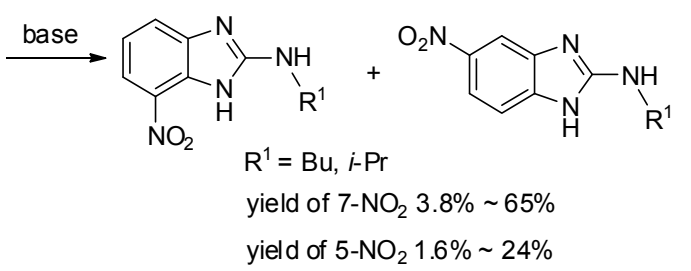

\section{Scheme 15}

这种合成方法中, 带有离去基团 $\left(\mathrm{R}^{3}=\mathrm{OR}\right.$, Chart 1) 的 $N$ 进攻芳环邻位碳, 得到的中间体在过量碱的协助下 消除 $\mathrm{ROH}$ 得到具有环外碳氮双键的产物并且随即芳环 化得到目标产物(Scheme 16) ${ }^{[84]}$.

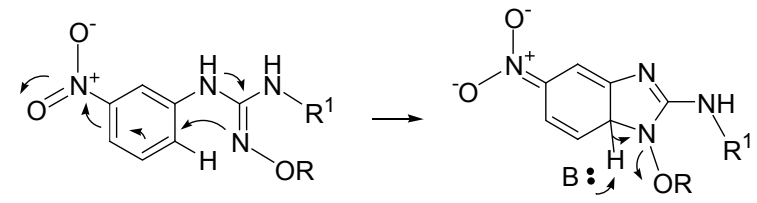<smiles>[R1]Nc1nc2cc([N+](=O)[O-])ccc2[nH]1</smiles>

Scheme 16

次氯酸钠和高价碘化合物在无过渡金属参与下可 以促进碳-氢键官能团化用于化学转化. 2013 年 Alla 等 ${ }^{[85]}$ 以 $m$-CPBA 为氧化剂在碘苯催化下由苯榺衍生物 合成苯并咪唑(Eq. 8).

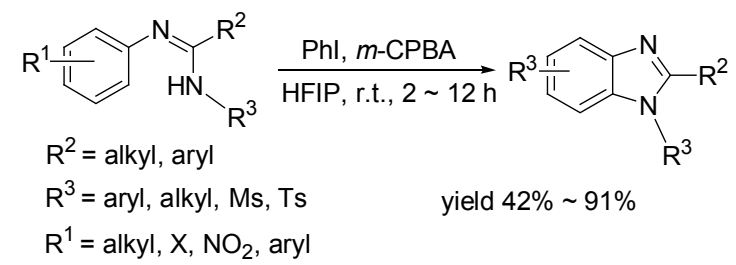

这种方法的反应机理和芳环的亲电取代反应机理 相似，首先中间体芳基朕在高碘试剂的作用下得到 $\mathrm{N}$ 被 碘代的化合物 15, 15 发生分子内芳环的亲电取代反应并 且形成碳一氮键, 再经过芳环化得到目标产物(Scheme 17). 由于是芳环的亲电取代反应，所以对某些不对称 芳基脒这个反应会有多个产物.

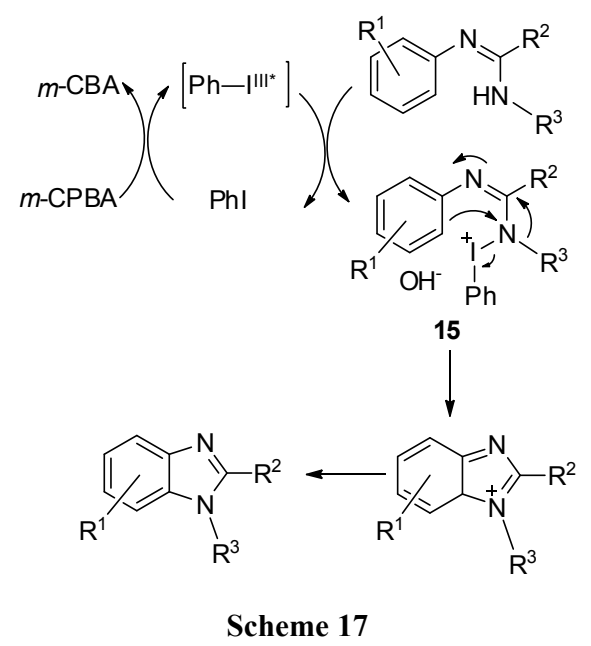

通过 Buchwald-Hartwig 反应构建 $\mathrm{C}-\mathrm{N}$ 键实现 $N$ 芳基化的方法常用来合成吲哚、吡啶吲哚和吡啶并苯并 咪唑环以及其他的杂环。同样，邻卤芳基脒衍生物 $\left(\mathrm{R}^{4}=\mathrm{X}\right.$, Chart 1) 通过这个反应发生 $N$-芳基化反应也被 用来合成苯并咪唑类化合物. 为了扩展 $\mathrm{R}^{2}$ 和 $\mathrm{R}^{3}$ (Chart 1)的范围, 2012 年 Sadig 等 ${ }^{[86]}$ 以邻卤芳基苯胺和各种羧 酸衍生物反应得到 $\mathrm{R}^{2}$ 不同的 $N$-烷基亚甲基邻卤芳基胺 
16,16 和不同的伯胺 $\mathrm{R}^{3} \mathrm{NH}_{2}$ 得到 $\mathrm{R}^{2}$ 和 $\mathrm{R}^{3}$ 不相同的邻卤 芳基苯脒 17 (Scheme 18), 并且在钯催化下发生 $N$-芳基 化反应得到苯并咪唑类化合物. 同时, 他们引入微波技 术，大大缩短了反应时间. 综合来看，该方法除了使用 了昂贵的钯催化剂和配体之外, 在反应适用性方面仍然 具有很大的优势.

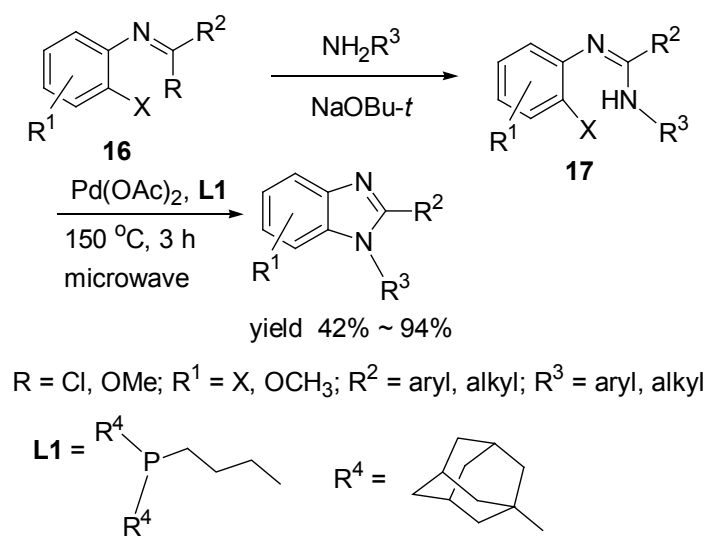

\section{Scheme 18}

尽管 Buchwald-Hartwig 反应在 C-N 键形成方面具 有很大的优势，但是由于配体不易得和钯金属昂贵制约 了它的应用, 而以金属铜作为催化剂催化构建 $\mathrm{C}-\mathrm{N}$ 键 则成为构建 $\mathrm{C}-\mathrm{N}$ 键的大趋势. 2012 年 $\mathrm{Yu}$ 等 ${ }^{[87]}$ 报道用 碘化亚铜催化邻溴苯胍的 $N$-芳基化反应合成 2-氨基苯 并咪唑(Eq. 9).

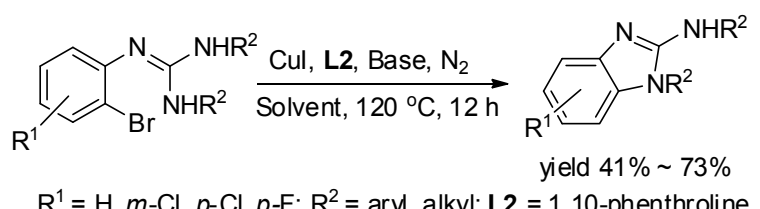

2008 年, Brasche 等 ${ }^{[88]}$ 在氧气中用醋酸铜催化苯榺 合成 2-取代苯并咪唑. 这种方法中, 苯脒首先和氧化态 的铜反应得到 $\mathrm{N}-\mathrm{Cu}$ 加成产物, 加成物可能经过 $\mathrm{a}$ 和 $\mathrm{b}$ 两种途径得到产物(Scheme 19).

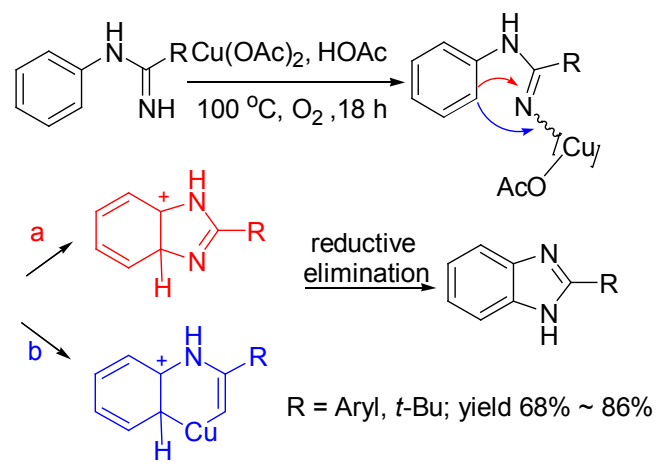

Scheme 19
2013 年, $\mathrm{Qu}$ 等 ${ }^{[89]}$ 以 $\mathrm{Cu}_{2} \mathrm{O}$ 催化脒化合物的盐酸盐和 邻卤苯胺反应合成邻氨基苯脒衍生物，再经过脱氨环合 得到苯并咪唑(Scheme 20).

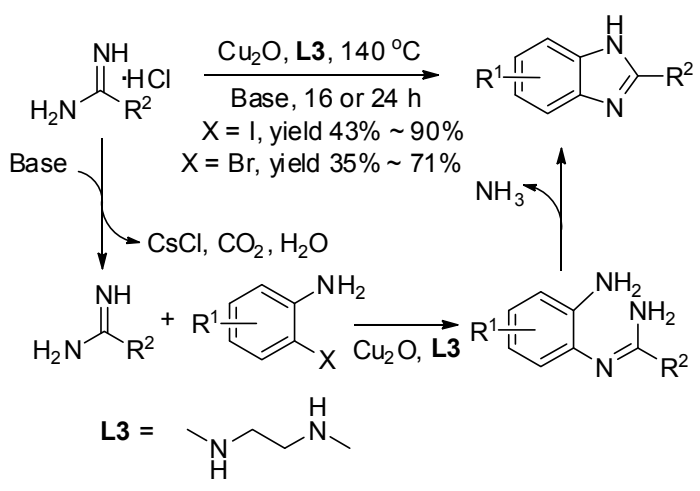

Scheme 20

2010 年 Peng 等 ${ }^{[90]}$ 用氧化亚铜催化邻卤苯脒分子内 $N$-芳基化反应合成 2-取代苯并咪唑(Eq. 10). 这种方法 中, 邻碘芳基榺的活性最高, 其次邻溴芳基脒, 邻氯芳 基脒的活性最低.

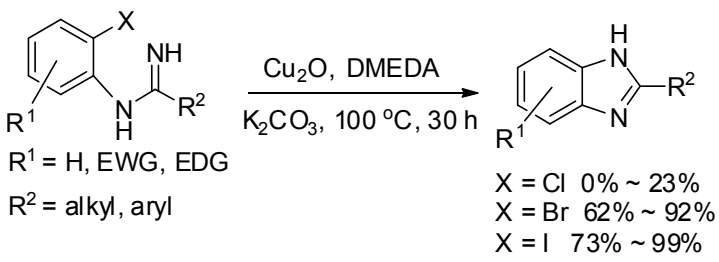

一般说来，在 Ullmann 反应中，首先一分子底物和 碱与铜形成 $\mathrm{Cu}-\mathrm{N}$ 键得到 $\mathrm{Cu}-\mathrm{N}$ 络合物(Scheme 21) ${ }^{[91]}$, 这个络合物发生分子内氧化加成一还原消除得到产物.

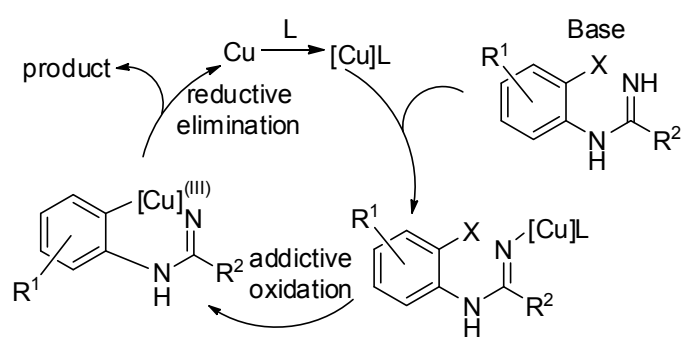

\section{Scheme 21}

2012 年 Liubchak 等 ${ }^{[92]}$ 用铜作为催化剂也合成了含 有苯并咪唑环的稠环. 他们以碘化亚铜催化含有并环的 脒，反应时间 $1 \sim 6 \mathrm{~h}$ ，反应收率在 $90 \%$ 以上，从而成功 地取代了昂贵的钯类金属催化剂并且构建了含有苯并 咪唑环的三环结构(Eq. 11).

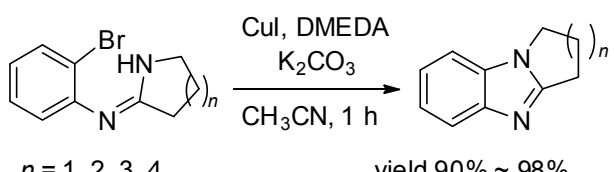


最近, Ullmann 反应自由基机理得到验证 ${ }^{[93]}$, 从此 除了 Buchwald-Hartwig 反应和 Ullmann 的氧化加成机理 构建 $\mathrm{C}-\mathrm{N}$ 键之外, 化学家们又有了构建 $\mathrm{C}-\mathrm{N}$ 的新利 器. 据报道, Ullmann 自由基机理在构建坐类 $\mathrm{C}-\mathrm{N}$ 成键 方面具有温度低(室温甚至 $-40{ }^{\circ} \mathrm{C}$ )收率高的优势 $(\mathrm{Eq}$. $12)^{[94]}$.

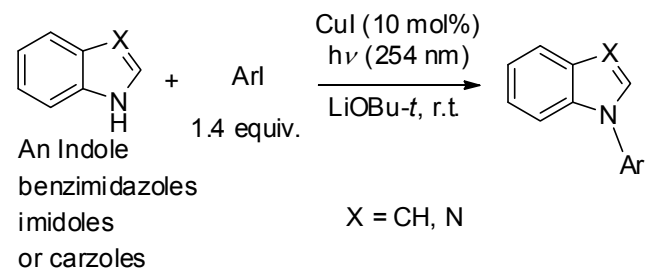

我们课题组一直以来致力于研究和开发金属催化 的高效和高原子经济性的反应, 并且一直以来对金催化 的简单高效反应感兴趣. 自从 2009 年以来, 我们 ${ }^{[95]}$ 研 究了金催化两类底物的加成反应, 一类是醇和磺酰胺等 $\mathrm{O}$ 或 $\mathrm{N}$ 亲核试剂对连烯的催化氢氧和氢氮加成反应 (Scheme 22).

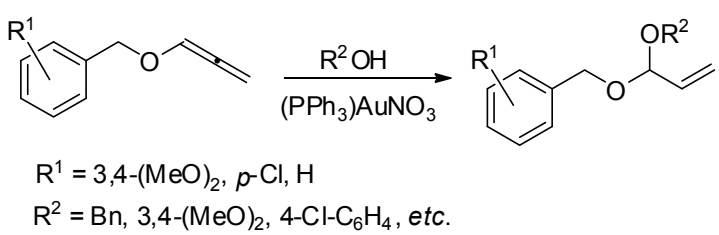

$$
\begin{aligned}
& \mathrm{R}_{\mathrm{R}^{1}=\mathrm{Ar} ; \mathrm{R}^{3}=\mathrm{Me}, \mathrm{Et}, \mathrm{Bn}, i-\mathrm{Pro}, t-\mathrm{Bu} \text {, cyclohexyl }}^{\mathrm{H}_{2} \mathrm{SO}_{4}}+\mathrm{R}^{3} \mathrm{OH} \underset{\mathrm{R}^{2}}{\stackrel{\left(\mathrm{PPh}_{3}\right) \mathrm{AuNO}_{3}}{\longrightarrow}} \mathrm{CH}_{3}
\end{aligned}
$$

\section{Scheme 22}

另一类是磺酸和磺酰胺等 $\mathrm{O}$ 或 $\mathrm{N}$ 亲核试剂对非活 性炔烃的催化氢氧和氢氮加成反应(Eq. 13) ${ }^{[96]}$. 这些金 催化的对重键的加成反应高效地构建了 $\mathrm{C}-\mathrm{N}$ 和 $\mathrm{C}-\mathrm{O}$ 键, 对构建杂环具有重要意义.

$$
\begin{aligned}
& \|_{\mathrm{R}^{1}}^{\mathrm{R}^{2}}+\mathrm{R}^{3} \mathrm{SO}_{3} \mathrm{H} \stackrel{[\mathrm{Au}]}{\longrightarrow} \mathrm{R}_{\mathrm{R}^{3} \mathrm{O}_{2} \mathrm{SO}} \\
\mathrm{R}^{1} & =\mathrm{Alkyl}-\mathrm{C}_{6} \mathrm{H}_{4}, \mathrm{X}_{-} \mathrm{C}_{6} \mathrm{H}_{4}, 4-\mathrm{CH}_{3} \mathrm{O}-\mathrm{C}_{6} \mathrm{H}_{4} \\
\mathrm{R}^{2} & =\mathrm{H}, \mathrm{CH}_{3}, \mathrm{C}_{6} \mathrm{H}_{5} \\
\mathrm{R}^{3} & =\mathrm{CH}_{3}, 4-\mathrm{Tol},
\end{aligned}
$$

除了以上两类外, 我们还研究了金催化连烯氧化脱水环合的反应，高收率地合成了苯并咪唑类化合物 (Scheme 23) ${ }^{[97]}$.

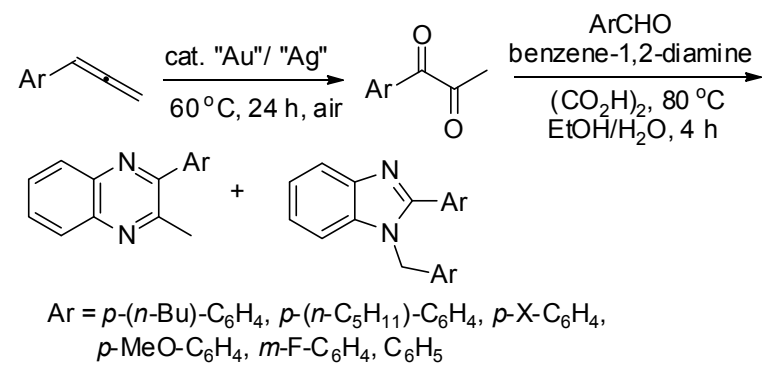

Scheme 23

基于我们实验室对金催化重键加成反应研究，我们 设想，在金催化剂端基炔烃能够和邻苯二胺反应得到中 间体 $N$-亚甲基邻苯二胺，该中间体在金存在的条件下 脱氢环合为苯并咪唑类化合物，从而实现金串联催化合 成苯并咪唑类化合物. 要实现该串联反应，首先要求金 催化端炔选择性氢胺化得到 18, 18 异构化为热稳定更好 的 19 (Eq. 14); 其次, 尽管金可以催化氧化烯烃和醇 等[98]，但是却没有文献报道过用 19 或者苯并咪唑啉在 金催化下氧化脱氢得到目标产物. 这方面的研究正在进 行中.

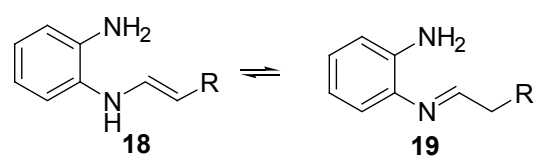

\section{4 结论和展望}

总之，第一次以合成苯并咪唑类化合物的三类中间 体为总体框架，整合了合成苯并咪唑类化合物的各种合 成方法. 通过这种归纳，我们可以将设计合成这类化合 物转化为设计路线合成这些中间体，中间体合成方法就 是新的合成苯并咪唑类化合物的方法. 比如我们已经可 以通过很多方法合成酰胺类化合物，其中过渡金属催化 合成酰胺类化合物就是一种，在 Chemical Reviews 上有 专门的综述. 这些方法的使用可以用其他原料替代醛, 从而扩展了底物范围，同时也实现了合成方法的多样 性. 再比如, 由于在半导体材料存在下, 醇类可以氧化 为醛，醛和胺反应得到 $N$-亚甲基邻苯二胺，而这正是合 成苯并咪唑类化合物的中间体. 2012 年科学家通过实验 证实 Ullmann 反应存在自由基机理，并且在很低温度反 应仍然可以发生，这个重大发现正在给古老的 Ullmann 反应注入新的活力; 另外, 方兴未艾的 $\mathrm{C}-\mathrm{H}$ 活化官能 才化也为人们高原子经济性地构建碳一杂键提供了前所 未有的利器，而以上这两个领域的进步无疑会促进从芳 基脒(胍)中间体合成苯并咪唑类化合物方法的发展．同 样不容忽视的还有新技术、新材料和负载技术等在有机 合成领域的应用，也将促进这类化合物合成方法的开 发. 


\section{References}

[1] Zhan, P.; Chen, X. W.; Li, D. Y.; Fang, Z. J.; De Clercq, E.; Liu, X. Y. Med. Chem. Commun. 2013, 33, S1, E1.

[2] Leoni, L. M. Semin. Hematol. 2011, 48, S4.

[3] Hranjec, M.; Starčević, K.; Pavelić, S. K.; Lučin, P.; Pavelić, K.; Karminski Zamola, G. Eur. J. Med. Chem. 2011, 46, 2274.

[4] (a) Shingalapur, R. V.; Hosamani, K. M.; Keri, R. S.; Hugar, M. H. Eur. J. Med. Chem. 2010, 45, 1753.

(b) Jain, P.; Sharma, P. K.; Rajak, H.; Pawar, R. S.; Patil, U. K.; Singour, P. K. Arch. Pharm. Res. 2010, 33, 971.

[5] (a) Sharma, M. C.; Kohli, D.; Sharma, S.; Sharma, A. D. Pelagia Res. Lib. 2010, 1, 104

(b) Shah, D. I.; Sharma, M.; Bansal, Y.; Bansal, G.; Singh, M. Eur. J. Med. Chem. 2008, 43, 1808 .

[6] Achar, K.; Hosamani, K. M.; Seetharamareddy, H. R. Eur. J. Med. Chem. 2010, 45, 2048.

[7] Kuş, C.; Ayhan-Kılcıgil, G.; Özbey, S.; Kaynak, F. B.; Kaya, M.; Çoban, T.; Can-Eke, B. Bioorg. Med. Chem. 2008, 16, 4294.

[8] Pérez-Villanueva, J.; Santos, R.; Hernández-Campos, A.; Giulianotti, M. A.; Castillo, R.; Medina-Franco, J. L. Med. Chem. Commun. 2011, 2, 44.

[9] Meng, J.-P.; Geng, R.-X.; Zhou, C.-H.; Gan, L.-L. Chin. J. New Drugs 2009, 18, 1505 (in Chinese).

(孟江平, 耿蓉霞, 周成合, 甘淋玲, 中国新药杂志, 2009, 18 , 1505.)

[10] Li, Y.; Ma, H.-Q.; Wang, Y.-L. Chin. J. Org. Chem. 2008, 28, 210 (in Chinese).

(李炎, 马会强, 王玉炉, 有机化学, 2008, 28, 210.)

[11] Chawla, A.; Kaur, G.; Sharma, A. K. Int. J. Pharm. Phytopharmacol. Res. 2012, 2, 148.

[12] Panda, S. S.; Malik, R.; Jain, S. C. Curr. Org. Chem. 2012, 16, 1905.

[13] Mao, Z.-Z.; Wang, Z.-Y.; Hou, X.-N.; Song, X.-M.; Luo, Y.-F. Chin. J. Org. Chem. 2008, 28, 542 (in Chinese). (毛郑州, 汪朝阳, 侯晓娜, 宋秀美, 罗玉芬, 有机化学, 2008, 28, 542.)

[14] Mao, Z.-Z.; Li, J.-X.; Xue, F.-L.; Chen, R.-H.; Wang, Z.-Y. Chem. Res. Appl. 2012, 24, 497 (in Chinese). (毛郑州, 李建晓, 薛福玲, 陈任宏, 汪朝阳, 化学研究与应用, 2012, 24, 497.)

[15] Chawla, A.; Kaur, R.; Goyal, A. J. Chem. Pharm. Res. 2011, 3, 925.

[16] Peng, P.; Xiong, J.-F.; Li, B.; Mo, G.-Z.; Chen, R.-H.; Wang, Z.-Y. Chin. J. Org. Chem. 2013, 33, 1891 (in Chinese). (彭湃, 熊金锋, 李豹, 莫广珍, 陈任宏, 汪朝阳, 有机化学, 2013, 33, 1891.)

[17] Carvalho, L. C.; Fernandes, E.; Marques, M. M. B. Chem.-Eur. J. 2011, 17, 12544.

[18] Mamedov, V. A.; Murtazina, A. M. Russ. Chem. Rev. 2011, 80, 397.

[19] (a) Dawood, K. M.; Elwan, N. M.; Farahat, A. A.; Abdel-Wahab, B. F. J. Heterocycl. Chem. 2010, 47, 2437.

(b) Dawood, K. M.; Elwan, N. M.; Abdel-Wahab, B. F. ARKIVOC 2011, (i), 111

(c) Dawood, K. M.; Abdel-Wahab, B. F. ARKIVOC 2010, (i), 333.

[20] Linschitz, H.; Grellmann, K. H. J. Am. Chem. Soc. 1964, 86, 303.

[21] Carvalho, L. C. R.; Fernandes, E.; Marques, M. M. B. Chem.-Eur. J. 2011, 17, 12544.

[22] Chebolu, R.; Kommi, D. N.; Kumar, D.; Bollineni, N.; Chakraborti, A. K. J. Org. Chem. 2012, 77, 10158.

[23] Das, B.; Holla, H.; Srinivas, Y. Tetrahedron Lett. 2007, 48, 61.
[24] Ghorbani Vaghei, R.; Veisi, H. Mol. Diversity 2010, 14, 249.

[25] Khosravi, K.; Kazemi, S. Chin. Chem. Lett. 2012, 23, 61.

[26] G Smith, J.; Ho, I. Tetrahedron Lett. 1971, 12, 3541.

[27] Lin, S. N.; Yang, L. H. Tetrahedron Lett. 2005, 46, 4315.

[28] Panda, S. S.; Jain, S. C. Synth. Commun. 2011, 41, 729.

[29] Zhang, C.; Zhang, L.-R.; Jiao, N. Green Chem. 2012, 14, 3273.

[30] Chen, G.-F.; Shen, H.-D.; Jia, H.-M.; Zhang, L.-Y.; Kang, H.-Y.; Qi, Q.-Q.; Chen, B.-H.; Cao, J.-L. Aust.J. Chem. 2013, 66, 262.

[31] Ben Alloum, A.; Bakkas, S.; Soufiaoui, M. Tetrahedron Lett. 1998, $39,4481$.

[32] Chang, J. B.; Zhao, K.; Pan, S. F. Tetrahedron Lett. 2002, 43, 951

[33] Beaulieu, P. L.; Haché, B.; von Moos, E. Synthesis 2003, 1683.

[34] Wang, H.-S.; Miao, J.-Y.; Zhao, L.-F. Chin. J. Org. Chem. 2005, 25, 615 (in Chinese).

(王宏社，苗建英，赵立芳，有机化学，2005, 25, 615.)

[35] (a) Osowska, K.; Miljanic, O. S. J. Am. Chem. Soc. 2011, 133, 724. (b) Lee, I. L.; Li, S.-R.; Chen, K.-F.; Ku, P.-J.; Singh, A. S.; Kuo, H.-T.; Wen, Y.-S.; Chu, C.-W. Eur. J. Org. Chem. 2012, 15, 2906.

[36] Sharghi, H.; Hosseini Sarvari, M.; Moeini, F. Can. J. Chem. 2008, $86,1044$.

[37] Bahrami, K.; Khodaei, M. M.; Naali, F. J. Org. Chem. 2008, 73, 6835.

[38] Chandrachood, P. S.; Garud, D. R.; Gadakari, T. V.; Torane, R. C.; Deshpande, N. R.; Kashalkar, R. V. Acta Chem. Slov. 2011, 58, 367.

[39] Sharghi, H.; Aberi, M.; Doroodmand, M. M. Adv. Synth. Catal. 2008, 350, 2380.

[40] Singh, M. P.; Sasmal, S.; Lu, W.; Chatterjee, M. N. Synthesis 2000 1380.

[41] Kadish, K. M.; Smith, K. M.; Guilard, R. The Porphyrin Handbook: Inorganic, Organometallic and Coordination Chemistry, Vol. 3, Elsevier, London, 2003.

[42] Abdollahi Alibeik, M.; Moosavifard, M. Synth. Commun. 2010, 40, 2686.

[43] Fazlinia, A.; Mosslemin, M. H.; Sadoughi, H. J. Korean Chem. Soc. 2010, 54, 579.

[44] Chen, G. F.; Dong, X. Y.; Meng, F. Z.; Chen, B. H.; Li, J. T.; Wang, S. X.; Bai, G. Y. Lett. Org. Chem. 2011, 8, 464.

[45] Leutbecher, H.; Constantin, M. A.; Mika, S.; Conrad, J.; Beifuss, U. Tetrahedron Lett. 2011, 52, 605.

[46] Coppola, G. M. Synth. Commun. 2008, 38, 3500.

[47] Chen, Y. X.; Qian, L. F.; Zhang, W.; Han, B. Angew. Chem., Int Ed. 2008, 120, 9470

[48] Rostamizadeh, S.; Amani, A. M.; Aryan, R.; Ghaieni, H. R.; Norouzi, L. Monatsh. Chem. 2009, 140, 547.

[49] Aliyan, H.; Fazaeli, R.; Fazaeli, N.; Mssah, A. R.; Naghash, H. J.; Alizadeh, M.; Emami, G. Heteroat. Chem. 2009, 20, 202.

[50] Chari, M. A.; Shobha, D.; Kenawy, E. R.; Al Deyab, S. S.; Reddy, B.; Vinu, A. Tetrahedron Lett. 2010, 51, 5195.

[51] Dhakshinamoorthy, A.; Kanagaraj, K.; Pitchumani, K. Tetrahedron Lett. 2011, 52, 69 .

[52] Meshram, G. A.; Patil, V. D. Int. J. Chem. Sci. 2010, 8, 119.

[53] Adharvana Chari, M.; Shobha, D.; Sasaki, T. Tetrahedron Lett. 2011, 52, 5575.

[54] Behbahani, F. K.; Ziaei, P.; Fakhroueian, Z.; Doragi, N. Monatsh. Chem. 2011, 142, 9, 901

[55] Zhu, G. M.; Zhuo, Z. J.; Cui, D. M.; Zhang, C. In Chinese Chemistry Society $\bullet$ The 8th National Organic Chemistry Conference \& the 1th Chongqing International Symposium on Organic Chemistry, Chongqing, 2013, p. 389 (in Chinese).

(朱观明, 卓子健, 崔冬梅, 张辰, 在中国化学会第八届有机化 学会议暨首届重庆有机化学国际研讨会, 重庆, 2013, p. 389.) 
[56] Han, X. M.; Ma, H. Q.; Wang, Y. L. ARKIVOC 2007, xiii, 150.

[57] Chakrabarty, M.; Mukherjee, R.; Karmakar, S.; Harigaya, Y. Monatsh. Chem. 2007, 138, 1279.

[58] Mohammadi, A. A.; Azizian, J.; Karimi, N. Heterocycles 2009, 78, 2337.

[59] Wang, X. B.; Jian, T. Y.; Ma, X. W.; He, L. Chin. J. Org. Chem. 2012, 32, 2181 (in Chinese). (王湘波，简腾跃，马晓伟，何林，有机化学, 2012, 32, 2181.)

[60] Srinivas, U.; Srinivas, C.; Narender, P.; Rao, V. J.; Palaniappan, S. Catal. Commun. 2007, 8, 107.

[61] Sharghi, H.; Asemani, O.; Khalifeh, R. Synth. Commun. 2008, 38, 1128.

[62] Saha, D.; Saha, A.; Ranu, B. C. Green Chem. 2009, 11, 733.

[63] Rostamizadeh, S.; Aryan, R.; Ghaieni, H. R.; Amani, A. M. J. Heterocycl. Chem. 2009, 40, 74.

[64] Lei, M.; Ma, L.; Hu, L. H. Synth. Commun. 2012, 42, 2981.

[65] Jadhav, G. R.; Shaikh, M. U.; Kale, R. P.; Gill, C. H. Chin. Chem. Lett. 2009, 20, 535.

[66] Bahrami, K.; Khodaei, M. M.; Nejati, A. Green Chem. 2010, 12, 1237.

[67] Molander, G. A.; Ajayi, K. Org. Lett. 2012, 14, 4242.

[68] (a) Dobereiner, G. E.; Crabtree, R. H. Chem. Rev. 2010, 110, 681. (b) Guillena, G.; Ramon, D. J.; Yus, M. Chem. Rev. 2010, 110, 1611 .

[69] Bala, M.; Kumar Verma, P.; Sharma, U.; Kumar, N.; Singh, B. Green Chem. 2013, 15, 1687.

[70] (a) Shiraishi, Y.; Sugano, Y.; Tanaka, S.; Hirai, T. Angew. Chem., Int. Ed. 2010, 49, 1656.

(b) Selvam, K.; Swaminathan, M. Tetrahedron Lett. 2011, 52, 3386.

[71] (a) Rope, M.; Isensee, R. W.; Joseph, L. J. Am. Chem. Soc. 1952, 74, 1095.

(b) Sandera, G.; Isensee, R. W.; Joseph, L. J. Am. Chem. Soc. 1954, $76,5173$.

[72] Clemens, J. J.; Davis, M. D.; Lynch, K. R.; Macdonald, T. L. Bioorg. Med. Chem. Lett. 2004, 14, 4903.

[73] Wen, X.; Bakali, J. E.; Deprez-Poulain, R.; Deprez, B. Tetrahedron Lett. 2012, 53, 2440.

[74] Thomas, O. D.; Soo, K. J. W. Y.; Peckham, T. J.; Kulkarni, M. P.; Holdcroft, S. J. Am. Chem. Soc. 2012, 134, 10753.

[75] Algul, O.; Kaessler, A.; Apcin, Y.; Yilmaz, A.; Jose, J. Molecules 2008, 13, 736 .

[76] Khattab, M.; Galal, S. A.; Ragab, F. A. F.; Diwani, H. I. E. Res. Chem. Intermed. 2013, 39, 2917.

[77] Khalili, S. B.; Sardarian, A. R. Monatsh. Chem. 2012, 143, 841.

[78] Bommegowda, Y. K.; Lingaraju, G. S.; Thamas, S.; Vinay Kumar,
K. S.; Pradeepa Kumara, C. S.; Rangappa, K. S.; Sadashiva, M. P. Tetrahedron Lett. 2013, 54, 2693.

[79] (a) Perry, R. J.; Wilson, B. D. J. Org. Chem. 1993, 58, 7016. (b) She, J.; Jiang, Z.; Wang, Y. Synlett 2009, 2023.

[80] VanVliet, D. S.; Gillespie, P.; Scicinski, J. J. Tetrahedron Lett. 2005, 46, 6741.

[81] Mamedov, V. A.; Zhukova, N. A.; Beschastnova, T. N.; Zakirova E. V. I.; Kadyrova, S. F.; Mironova, E. V.; Nikonova, A. G.; Latypov, S. K. Tetrahedron Lett. 2012, 53, 292.

[82] Mamedov, V. A.; Murtazina, A. M. Russ. Chem. Rev. 2011, 80, 397.

[83] Esser, F.; Ehrengart, P.; Ignatow, H. P. J. Chem. Soc., Perkin Trans. 1 1999, 1153.

[84] Makosza, M.; Winiarski, J. Acc. Chem. Res. 1987, 20, 282.

[85] Alla, S. K.; Kumar, R. K.; Sadhu, P.; Punniyamurthy, T. Org. Lett. 2013, 15, 1334.

[86] Sadig, J. E. R.; Foster, R.; Wakenhut, F.; Willis, M. C. J. Org. Chem. 2012, 77, 9473.

[87] Yu, H.; Liu, Q.; Li, Y.; Ni, C. Tetrahedron Lett. 2012, 53, 5253.

[88] Brasche, G.; Buchwald, S. L. Angew. Chem., Int. Ed. 2008, 120, 1958.

[89] Qu, Y. Y.; Pan, L.; Wu, Z. Q.; Zhou, X. G. Tetrahedron 2013, 69, 1717.

[90] Peng, J. S.; Ye, M.; Zong, C. J.; Hu, F. Y.; Feng, L. T.; Wang, X. Y.; Wang, Y. F.; Chen, C. X. J. Org. Chem. 2010, 76, 716.

[91] Giri, R.; Hartwig, J. F. J. Am. Chem. Soc. 2010, 132, 15860.

[92] Liubchak, K.; Nazarenko, K.; Tolmachev, A. Tetrahedron 2012, 68, 2993.

[93] Creutz, S. E.; Lotito, K. J.; Fu, G. C.; Peters, J. C. Science 2012, $338,647$.

[94] Ziegler, D. T.; Choi, J.; Muñoz-Molina, J. M.; Bissember, A. C.; Peters, J. C.; Fu, G. C. J. Am. Chem. Soc. 2013, 135, 13107.

[95] (a) Zhang, C.; Zhang, S. Q.; Cai, H. J.; Cui, D. M. Beilstein J. Org. Chem. 2013, 9, 1045.

(b) Cui, D. M.; Zheng, Z. L.; Zhang, C. J. Org. Chem. 2009, 74, 1426.

(c) Cui, D. M.; Zhuang, D. W.; Chen, Y.; Zhang, C. Beilstein J. Org. Chem. 2011, 7, 860

(d) Cui, D. M.; Yu, K. R.; Zhang, C. Synlett 2009, 1103.

[96] (a) Cui, D. M.; Zheng, J. Z.; Yang, L. Y.; Zhang, C. Synlett 2010, 809.

(b) Cui, D. M.; Meng, Q.; Zheng, J. Z.; Zhang, C. Chem. Commun. 2009, 1577.

[97] Cui, D.-M.; Zhuang, D.-W.; Chen, Y.; Zhang, C. Beilstein J. Org. Chem. 2011, 7, 860.

[98] Li, Z.-G.; Brouwer, C.; He, C. Chem. Rev. 2008, 108, 3239.

(Zhao, C.) 